\begin{tabular}{|c|c|}
\hline Title & Age related glomerular lesions with al buminuria in male cotton rats \\
\hline Author(s) & $\begin{array}{l}\text { Ichii, O samu; Nakamura, Teppei; Irie, Takao; Otani, Y uki; Hosotani, Marina; Masum, Md A bdul; Islam, Rashedul Md; } \\
\text { Horino, T aro; Sunden, Y uji; Elewa, Y aser Hosny A Ai; Kon, Y Ysuhiro }\end{array}$ \\
\hline Citation & $\begin{array}{l}\text { Histochemistry and Cell Biology, 153(1), 27-36 } \\
\text { https://doi.org/10.1007/s00418-019-01824-1 }\end{array}$ \\
\hline Issue Date & $2020-01$ \\
\hline Doc URL & http:/hdl.handle.net/2115/80101 \\
\hline Rights & $\begin{array}{l}\text { This is a post-peer-review, pre copy edit version of an article published in Histochemistry and Cell Biol ogy. The final } \\
\text { authenticated version is avail able online at: } h \text { ttp://dx.doi.org } / 10.1007 / 300418-019-01824.1\end{array}$ \\
\hline Type & article (author version) \\
\hline File Information & manuscript.pdf \\
\hline
\end{tabular}

Instructions for use 


\section{Age-related glomerular lesions with albuminuria in male cotton rats}

2

3 Osamu Ichii ${ }^{1}$, Teppei Nakamura ${ }^{1,2}$, Takao Irie $^{3}$, Yuki Otani ${ }^{1}$, Marina Hosotani ${ }^{1,4}$, Md. Abdul Masum ${ }^{1}$,

4 Rashedul Md. Islam ${ }^{1}$, Taro Horino ${ }^{5}$, Yuji Sunden ${ }^{6}$, Yaser Hosny Ali Elewa ${ }^{1,7}$, Yasuhiro Kon ${ }^{1}$

$6{ }^{1}$ Laboratory of Anatomy, Department of Basic Veterinary Sciences, Faculty of Veterinary Medicine,

$7 \quad$ Hokkaido University, Sapporo, Hokkaido, Japan

82 Section of Biological Science, Chitose Laboratory, Japan Food Research Laboratories, Chitose,

9 Hokkaido, Japan

${ }^{3}$ Medical Zoology Group, Department of Infectious Diseases, Hokkaido Institute of Public Health, Sapporo, Hokkaido, Japan

${ }^{4}$ Laboratory of Anatomy, School of Veterinary Medicine, Rakuno Gakuen University, Ebetsu, Hokkaido,

13 Japan

$14{ }^{5}$ Department of Endocrinology, Metabolism and Nephrology, Kochi Medical School, Kochi University, 15 Nankoku, Kochi, Japan

$16{ }^{6}$ Laboratory of Veterinary Pathology, Faculty of Agriculture, Tottori University, Tottori, Japan

$17{ }^{7}$ Department of Histology and Cytology, Faculty of Veterinary Medicine, Zagazig University, Zagazig, 18 Egypt

19 Tel \& Fax: +81-11-706-5189; E-mail: $\underline{\text { ichi-o@vetmed.hokudai.ac.jp }}$ 
Ichii $O$ et al. Glomerulus of aged cotton rats

25 Running headline: Glomerulus of aged cotton rats

26 Keywords: Cotton rats, aging, male, glomerulus, albuminuria, glomerular basement membrane, podocyte 27 


\section{Abbreviations}

29 1. chronic kidney disease (CKD)

$30 \quad$ 2. glomerular basement membrane (GBM)

$313 . \quad$ blood urea nitrogen (BUN)

32 4. serum creatinine $(\mathrm{sCr})$

$33 \quad$ 5. white blood cell (WBC)

$34 \quad 6 . \quad$ red blood cell (RBC)

35 7. hemoglobin (Hgb)

36 8. hematocrit (HCT)

37 9. sodium dodecyl sulfate-polyacrylamide gel electrophoresis (SDS-PAGE)

38 10. urinary creatinine $(\mathrm{uCr})$

39 11. periodic acid Schiff (PAS)

40 12. periodic acid methenamine silver (PAM)

41 13. phosphate-buffered saline (PBS) 


\section{Abstract}

43 The increased prevalence of aging-related chronic kidney disease (CKD) among humans is a problem

44 worldwide. Aged cotton rats (Sigmodon hispidus) are considered novel model animals for studying CKD, especially as the females develop severe tubulointerstitial lesions with anemia. To investigate the renal pathologic features in aged male cotton rats and their characteristic glomerular injuries, the animals were divided into young, adult, old-aged, and advanced-aged groups (1-4, 5-8, 9-12, and 13-17 months, respectively) and pathologically analyzed. Anemia and renal dysfunction, as indicated by hematologic and serologic parameters, were significantly milder in the advanced-aged males than in the old-aged females. The males had increased urinary albumin-to-creatinine ratios from the old-age period, with the advanced-aged males having significantly higher levels than those in the old-aged females and young males. The old-aged females did not show clear glomerular injuries, whereas the advanced-aged males showed membranous lesions characterized by irregular and thickened glomerular basement membranes (GBMs). Characteristically, several large-sized projections from the GBM toward the podocytes were observed by microscopy, and podocytes covering these projections effaced their foot processes. The advanced-aged males showed aging-related IgG immune-complex depositions in the paramesangial regions and along the GBM. Furthermore, the positive reaction for podocin (a podocyte molecule) was granulated along the GBM. Thus, we clarified the albuminuria associated with altered glomerular structures in advanced-aged cotton rats, and that these phenotypes were closely associated with aging. These data help to clarify the aging-related pathogenesis of glomerular injury.

61 


\section{Introduction}

Chronic kidney disease (CKD) is becoming a common and serious problem encountered in both human and veterinary medicine, given the growing trend toward the aging of society and the fact that the disease is associated with an older age in mammals (Bartges 2012; Coresh et al. 2007). The translational research based on Zoobiquity (Evans and Leighton 2014), targeting the elucidation of cross-species risk factors and pathogenesis, would be helpful in solving this common problem among mammalian species. In addition to genetic factors, several systemic factors also affect CKD development, such as infection, hypertension, and/or autoimmune disease-related conditions (Levey and Coresh 2012). Notably, in the Japanese people, glomerular diseases such as chronic glomerulonephritis, diabetic nephropathy, and renal sclerosis are the primary causes of CKD requiring dialysis (Masakane 2015), where men in particular tend to show a more rapid progression of the disease than women do (Silbiger and Neugarten 1995). Interestingly, in the veterinary area, the pathologic features of CKD differ among companion animals. For example, the glomerulus tends to be injured in dogs, whereas the tubulointerstitium is more often damaged in cats (Ichii et al. 2011).

The mammalian glomerulus has a crucial role in maintaining the glomerular filtration barrier. Structural disruption of the glomerulus causes proteinuria, a condition in which the urine is composed mainly of leaked serum albumin (hence called albuminuria). Among the cells that make up the glomerulus, the podocytes (i.e., glomerular epithelial cells) are important for the physiologic and pathologic regulation of the glomerular filtration barrier through their creation of a slit diaphragm, and for the maintenance of the glomerular basement membrane (GBM) together with the glomerular endothelial cells (Daehn 2018). The morphofunctional alteration of the glomerular filtration barrier critically affects the progression of CKD. In humans, the degree of proteinuria is closely and positively correlated with the increased incidence of end-stage renal failure (Iseki et al. 2003). Furthermore, the severity of proteinuria is negatively correlated with the survival scores both in dogs and cats (Rudinsky et al. 2018; Syme et al. 
2006). To facilitate the future development of novel diagnostic and/or therapeutic strategies for CKD, genetically modified animals in which functional molecules of the podocyte or the induction of anti-glomerulus immunity are targeted have been mainly used to clarify the molecular pathogenesis of albuminuria (Yang et al. 2018), as spontaneous animal models of this disease are scarce.

The cotton rat (Sigmodon hispidus) is an experimental rodent originating from the southern United States. Many studies have reported that this rodent is associated with an increased susceptibility to pathogenic human viruses, protozoans, metazoans, bacteria (e.g., Leishmania and Echinococcus), and respiratory disease viruses (Blanco et al. 2014). In addition, unique disease phenotypes have been identified in cotton rats, including fragile tails, stomach cancer, and cardiomyopathy (Faith et al. 1997). In our previous studies, we had also identified some unique phenotypes of this species, such as the presence of pharyngeal pouch remnants, visceral adipose inflammation and ectopic fat accumulation in the pancreas without obesity, and the predominance of CKD in the females (Ichii et al. 2016, 2018; Nakamura et al. 2018, 2019). Furthermore, we clarified that CKD in aged female cotton rats was more severe with aging and strongly affected by female sex hormones and the development of pyometra (Ichii et al. 2018). The main renal histologic feature of female cotton rats is chronic tubulointerstitial nephritis characterized by the infiltration of immune cells and the dilation of distal tubules rather than glomerular lesions, whereas the males show only moderate tubulointerstitial lesions (Ichii et al. 2016, 2018). Furthermore, although glomerular lesions are also a major pathologic feature of CKD (Japanese Society for Dialysis Therapy 2015), their detailed features and change of the functions associated with the glomerulus, including membranoproliferative lesions, immune-complex deposition, and proteinuria, are still unclear.

In this study, we carefully examined the glomerular histopathology and identified the glomerular lesions characterized by GBM abnormality with albuminuria in male cotton rats of advanced age. Additionally, the renal dysfunction indicated by the increases in blood urea nitrogen (BUN) and serum 
creatinine (sCr) levels was noted in aged females but not in males, although the males showed glomerular injury. Thus, this study suggested that cotton rats would be a suitable animal model for the study of spontaneous albuminuria with morphofunctional alteration of the GBM due to aging.

\section{Materials and methods}

\section{Animals}

117 The animal experimentation was performed according to the guidelines of the Hokkaido Institute of 118 Public Health (Sapporo, Japan) (Approval No.: K27-03). Male and female cotton rats (1-21 months of 119 age) were maintained as the HIS/Hiph strain through continuous inbreeding under conventional conditions at the Hokkaido Institute of Public Health. The animals were divided into four groups by age; namely, young (1-4 months of age), adult (5-8 months of age), old-aged (9-12 months of age), and advanced-aged (13-17 months of age). With the animals under deep isoflurane anesthesia, blood was collected from the vena cava, following which the animals were euthanized by severing of the abdominal aorta. The extracted serum was used for serologic analysis. The kidneys were fixed in $4 \%$ paraformaldehyde or in a $2 \%$ paraformaldehyde and $2.5 \%$ glutaraldehyde mixture for histopathologic analysis, and a part of the kidney was stored in Michel’s Transport Medium (Polysciences, Warrington, PA, USA).

Blood examination Hematologic analysis was performed using an XT-1800xi instrument (Sysmex, Kobe, Japan) for determination of the white blood cell (WBC) and red blood cell (RBCs) numbers, as well as the hemoglobin (Hgb) and hematocrit (HCT) values. For the serologic tests, the BUN and sCr levels were analyzed using a Fuji Dri-Chem 7000v analyzer (Fujifilm, Tokyo, Japan). 
Freshly voided urine was collected during 9 am and 5 pm on two separate days and pooled for urinalysis.

137 The urinary albumin was analyzed by sodium dodecyl sulfate-polyacrylamide gel electrophoresis

138 (SDS-PAGE). Approximately $10 \mu \mathrm{g}$ of protein per sample was separated by SDS-PAGE at $225 \mathrm{~V}$ for 40

139 min on Novex 4-20\% Tris-Glycine Mini Gels (Thermo Fisher Scientific, Waltham, MA, USA).

140 Molecular weight markers ranging from 6.5 to $200 \mathrm{kDa}$ (Bio-Rad, Hercules, CA, USA) and $0.5 \mu \mathrm{g}$ of

141 bovine serum albumin were included in each gel. The gels were stained with a Coomassie dye (GelCode

142 Blue Stain Reagent, Thermo Fisher Scientific) and analyzed using image analysis software (CS Analyzer;

143 ATTO, Tokyo, Japan). The urinary creatinine (uCr) level was measured by the alkaline picrate method

144 (Creatinine Companion Kit; Exocell, Philadelphia, PA, USA) and used for the normalization of the urinary albumin level.

\section{$147 \quad$ Histopathologic analysis}

148 The paraffin-embedded kidney sections were stained with periodic acid Schiff (PAS) or periodic acid 149 methenamine silver (PAM) for histopathologic analysis. Immunohistochemistry for podocin was

150 performed to evaluate the slit diaphragm feature. In brief, the sections were deparaffinized, heated in 151 Tris- $\mathrm{HCl}$ buffer (pH 9.0) for $15 \mathrm{~min}$ at $110^{\circ} \mathrm{C}$, and incubated with anti-human podocin rabbit antibody 152 (1:400; IBL, Gunma, Japan) followed by anti-rabbit IgG antibody (undiluted; Nichirei, Tokyo, Japan), 153 according to a previously published streptavidin-biotin method (Ichii et al. 2016). The color was 154 developed by incubating the sections in a 3,3'-diaminobenzidine tetrahydrochloride-hydrogen peroxide 155 solution. The same concentration of normal rabbit IgG (Santa Cruz Biotechnology, Dallas, TX USA) was used as a primary antibody control to confirm whether there were any specific reactions from using this antibody. 

washed with $0.01 \mathrm{M}$ phosphate-buffered saline (PBS) and embedded into a Tissue-Tek O.C.T. compound (Sakura Finetek, Tokyo Japan) with liquid nitrogen and isopentane. Frozen sections of 4- $\mu \mathrm{m}$ thickness were prepared and air-dried completely. The sections were then incubated with either chicken anti-cotton rat IgG antibody (1:3200; Immunology Consultants Laboratory, Portland, OR, USA) or rabbit anti-mouse IgA antibody (1:3200; Bethyl Laboratories, Montgomery, AL, USA) at $4^{\circ} \mathrm{C}$ overnight. A previous study

164 had suggested that there was cross-reactivity between mouse IgA and cotton rat IgA (Guichelaar et al. 165 2014). Then, the sections were washed with PBS and incubated with Alexa Fluor 488-conjugated donkey 166 anti-chicken IgY antibody (1:500; Jackson ImmunoResearch Laboratories, West Grove, PA, USA) and 167 Alexa Fluor 488-conjugated donkey anti-rabbit IgG antibody (1:500; Thermo Fisher Scientific) at room temperature for $30 \mathrm{~min}$. After a wash with PBS, the sections were mounted and examined using a BZ-X710 microscope (Keyence, Osaka, Japan). glutaraldehyde mixture for $4 \mathrm{~h}$, then post-fixed in $1 \%$ osmium tetroxide for $2 \mathrm{~h}$, and finally embedded in Quetol 812 resin (Nisshin EM, Tokyo, Japan). Ultrathin sections were then doubly stained with uranyl acetate and lead citrate and observed using a JEM-1400 Plus microscope (JEOL, Tokyo, Japan).

176 The results are expressed as the median value. The Mann-Whitney U test was used to compare two groups $(P<0.05)$. The Kruskal-Wallis test was used for comparing more than three populations, and multiple comparisons were performed using Dunnett's test when a significant difference was observed $(P$ $<0.05)$. Spearman's correlation test $(P<0.05)$ was used to analyze the correlation between two parameters. 


\section{Results}

183

184

185

186

Anemia and tubulointerstitial lesions are milder in males than in females

We had already previously clarified that female cotton rats developed renal anemia (Ichii et al. 2016). In this study, we also examined anemic parameters in the males and compared them with those of the females (Fig. 1). As in our previous study, the RBC, HCT, and Hgb levels in the females had decreased with aging (Fig. 1a-c). In the males, the RBC and Hgb levels were significantly more decreased in the advanced-aged group than in the other groups (Fig. 1a and c), but the HCT levels were not significantly changed among the groups (Fig. 1b). The RBC, HCT, and Hgb values tended to be higher in the males than in the females, and significant sex-related differences were observed in the adult and old-aged groups. When we compared these anemic parameters between the advanced-aged males and old-aged females, the former showed significantly higher values for all parameters $(P<0.001)$. In contrast, no clear age-related change was observed in the WBC counts of both sexes, but the old-aged female group showed significantly higher values than those of the old-aged and advanced-aged male groups (Fig. 1d).

Our previous study had indicated that renal anemia in cotton rats seemed to be strongly affected by the tubulointerstitial lesion (Ichii et al. 2016). As shown in Fig. 1e, the females developed severe tubulointerstitial inflammation with dilation of the renal tubule at the corticomedullary junction, whereas these lesions were clearly milder in the males, even in the advanced-aged group (Fig. 1e).

\section{Aged males developed albuminuria}

Fig. 2 shows the renal functional parameters. The BUN levels tended to increase with aging in both sexes, and the females showed significantly higher values than those of the males in the old-aged groups (Fig. 2a). Furthermore, the advanced-aged males showed significantly higher values relative to those of the young and old-aged groups. The sCr levels tended to be increased with aging in the females rather than in 
the males, with the old-aged and advanced-aged female groups showing significantly higher values (Fig. 2b).

Next, we used SDS-PAGE to examine the urinary albumin excretion levels in the males (Fig. 2c). As shown in the figure panels, the clear band detected at approximately $66 \mathrm{kDa}$ indicated that the albumin (and its band intensity) level tended to be higher in the aged males. With regard to the quantified urinary albumin-to-creatinine ratio (uACR), the values increased with aging in the males, where the advanced-aged males showed significantly higher values than those of the young males $(P<0.001$, Fig. 2d). Furthermore, the advanced-aged males also showed significantly higher values than those of the old-aged females. As shown in Fig. 2e, the uACR was significantly and positively correlated with age in the males $(\rho=0.804, P<0.001)$.

217 Fig. 3 shows the glomerular histopathologic features in the cotton rats. Males of young age had a normal glomerulus structure, where no proliferative or membranous lesions were observed (Fig. 3a). Furthermore, as reported previously (Ichii et al. 2016), the females in all the age groups examined also showed no clear glomerular lesions, but cell infiltrations with fibrous lesions were observed around the renal corpuscles (Fig. 3b). In contrast, the aged males manifested glomerular lesions that were characterized by thickened or wrinkled GBMs in the PAS-stained sections, and these lesions were clearer in the advanced-aged group (Fig. 3c and d). Compared with the GBM alterations, proliferative lesions were scarce or mild in the advanced-aged males. Interestingly, in this advanced-aged group of males, the high-magnification image showed PAS-positive rod-like structures along the GBM that were projected toward the podocytes nephropathy. 
231 As shown in Fig. 4a, the young male cotton rats did not show any positive reactions for IgG or IgA in the immunofluorescence assay using snap-frozen sections. In contrast, the advanced-aged males showed positive reactions for IgG, but not for IgA, which were observed mainly in the paramesangial regions and along the GBM (Fig. 4b). Fig. 4c shows the localization of podocin, a slit diaphragm molecule, where a positive reaction was clearly observed along the GBM in the young group of males. In contrast, these reactions appeared as granulated patterns of various sizes in the advanced-aged males.

Males showed age-related alteration of the GBM

Fig. 5 shows the ultrastructure of the glomerulus in the advanced-aged group of male cotton rats. Similar to the light microscopy observations, projections from the GBM toward the podocytes were clearly

241 observed (Fig. 5a and b). Furthermore, the thickness of the GBM differed among the glomerular capillary 242 rete, being either thin (Fig. 5c) or thickened with large-sized projections (Fig. 5d). In the latter case, the

243 foot processes of the podocytes covering the GBM were effaced. In the other lesions, the incorporation of

244 the mesangial matrix into the paramesangial regions (Fig. 5e) and the reticular pattern of the GBM (Fig.

245 5f) were also observed. 


\section{Discussion}

In this study, we found that advanced-aged male cotton rats developed albuminuria without severe renal dysfunction, as indicated by the remarkable elevations of BUN or sCr. As previously shown in aged rodents, including mice and rats, albuminuria was generally observed with glomerular injury and reduction of the glomerular filtration rate (Alt et al. 1980; Lim et al. 2012). As a common feature between aged humans and animals, the kidneys show membranous lesions characterized by GBM thickening and wrinkling associated with the loss of the capillary loops, as well as proliferative lesions, such as mesangial matrix expansion due to the imbalanced production of extracellular matrixes, and these age-related glomerular changes are more apparent in males than in females (Anderson and Brenner, 1986; Zhou et al. 2008). Although cotton rats seemed to develop the same age-related kidney damage as found in other rodent species, clear sex-related differences were characteristically observed in their phenotypes. In brief, whereas aged female cotton rats developed severe renal inflammation with renal anemia and renal dysfunction (Ichii et al. 2016, 2018), these phenotypes were milder in male cotton rats. Furthermore, we had also already clarified that pyometra, female sex hormone changes, and induction of the estrogen receptor in the renal tubules regulated the tubulointerstitial lesions in female cotton rats (Ichii et al. 2018). These CKD-associated phenotypes found in the females were inhibited by ovariectomy, indicating the

264 exacerbative activity of the female sex hormones toward the tubulointerstitial lesions (Ichii et al. 2018). In

265 addition, we examined the effects of male sex hormones on the development of albuminuria in advanced-aged males by castrating the cotton rats at 2 months before sampling (at $~ 10.5$ months of age, $n$ =4). As a result, because there was no change in the albuminuria by castration (Supplemental Fig. S1), we surmised that the male sex hormones contributed poorly to the phenotype observed in the advanced-aged males. Therefore, the glomerular damage in the male cotton rats was strongly affected by aging, whereas the renal pathogenesis in the female cotton rats was more strongly affected by the female sex hormones or reproductive organ abnormalities, as we had reported previously. 
In general, morphofunctional changes of the glomerular filtration barrier cause leakage of the serum protein into primarily the urine, resulting in albuminuria (Anderson and Brenner. 1986; Yumura et al. 1989). From the observed histopathologic features of the glomerular filtration barrier in advanced-aged male cotton rats, we clarified the presence of 1) GBM alterations, 2) the deposition of IgG-related immune complexes, and 3) podocyte injuries. As commonly found among aged rodents (Yumura et al. 1989), the deposition of immune complexes (particularly IgG) into the glomerulus tends to increase with aging. Furthermore, the GBM of the advanced-aged males showed no immune-mediated lesions, such as spike-like structures and double contouring, or mesangial proliferative lesions like those found in membranous nephropathy and proliferative glomerulonephritis. Therefore, the IgG depositions found in the glomerulus of the advanced-aged males would not be immune-mediated glomerular lesions but aging-related ones, as commonly found in other rodent species. Therefore, we concluded that the direct causal factors of albuminuria in the advanced-aged male cotton rats were the changes in podocyte morphology characterized by foot process effacement and altered expression of the slit diaphragm protein podocin, as well as GBM alterations. podocytes and random thickness, were characteristic features in the glomerulus of the advanced-aged male cotton rats. Importantly, in these lesions, effacement of the podocyte foot processes was clearly observed. Interestingly, the large projections of the GBM toward the podocytes were clearly different from the "spike lesions" found in the immune-complex depositions in the GBM. However, there are no clear reports related to these large-sized GBM projections in humans or in other laboratory rodents. The GBM is coordinately produced and maintained by the podocytes and glomerular endothelial cells, and is mainly composed of laminin, type IV collagen, nidogen, and heparan sulfate proteoglycan (Miner 2012).

294 In particular, Abrahamson et al. (2009) indicated the importance of the podocytes for the production of collagen $\alpha 3 \alpha 4 \alpha 5$ (IV). Therefore, there is a possibility that cotton rats might have a genetic deficiency 
(e.g., Alport syndrome) associated with the production and regulation of the GBM components. However, we surmise that these genetic variants or mutations would be less likely to contribute to the development of GBM abnormality in this species, because cotton rats do not develop GBM abnormality from a young age, as found in genetic mutation diseases, whereas the advanced-aged individuals do, especially the males. In fact, there are no data on the development of hearing loss and eye abnormalities in these rats, as found in patients with Alport syndrome. Furthermore, altered systemic conditions such as high-pressure and/or diabetic conditions also affect the morphologic alteration of the GBM. In fact, we had already reported that aged cotton rats develop metabolic disorders associated with visceral adipose inflammation and fatty pancreas without obesity, characterized by hyperinsulinemia, hyperglycemia, and dyslipidemia (Nakamura et al. 2019). Therefore, the histopathologic features of the GBM abnormality in the advanced-aged male cotton rats would be formed by several complex factors, including aging, genetic factors, and/or altered systemic conditions. of age-related injuries of the glomerular filtration barriers in humans and other animals.

314 Funding information This work was supported by JSPS KAKENHI (Grant No.: JP18K0703708).

318 Conflict of interest All the authors of this paper have no conflicts of interest to declare. 
Ichii $O$ et al. Glomerulus of aged cotton rats

320 Statement of human and animal rights The animal experimentation was performed according to the

321 guidelines of the Hokkaido Institute of Public Health, Sapporo, Japan (Approval No.: K27-03).

322

323 Author contributions

324 O.I., T.N., T.I., T.H., Y.H.A.E., and Y.K. conceived and designed the experiments. O.I., T.N., T.I., Y.O.,

325 M.H., M.A.M., and R.M.I. performed the experiments. O.I., T.N., and Y.S. analyzed the data. O.I. and

326 Y.K. wrote the paper.

327 


\section{References}

Abrahamson DR, Hudson BG, Stroganova L, Borza DB, St John PL (2009) Cellular origins of type IV

330 collagen networks in developing glomeruli. $J$ Am Soc Nephrol 20:1471-1479.

331 https://doi.org/10.1681/ASN.2008101086

332 Alt JM, Hackbarth H, Deerberg F, Stolte H (1980) Proteinuria in rats in relation to age-dependent renal changes. Lab Anim 14:95-101. https://doi.org/10.1258/002367780780942809

334 Anderson S, Brenner BM (1986) Effects of aging on the renal glomerulus. Am J Med 80:435-442.

335 https://doi.org/10.1016/0002-9343(86)90718-7

336 Bartges JW (2012) Chronic kidney disease in dogs and cats. Vet Clin North Am Small Anim Pract 42:669-692. vi. https://doi.org/10.1016/j.cvsm.2012.04.008

Blanco JC, Boukhvalova MS, Perez DR, Vogel SN, Kajon A (2014) Modeling human respiratory viral infections in the cotton rat (Sigmodon hispidus). $J$ Antivir Antiretrovir 6:40-42. https://doi.org/10.4172/jaa.1000093

341 Coresh J, Selvin E, Stevens LA, et al (2007) Prevalence of chronic kidney disease in the United States. JAMA 298:2038. https://doi.org/10.1001/jama.298.17.2038

343 Daehn IS (2018) Glomerular endothelial cells stress and cross-talk with podocytes in the development of

344 diabetic kidney disease. Front Med 5:76. https://doi.org/10.3389/fmed.2018.00076

345 Evans BR, Leighton FA (2014) A history of One Health. Rev Sci Tech 33:413-420.

346 Faith RE, Montgomery CA, Durfee WJ, Aguilar-Cordova E, Wyde PR (1997) The cotton rat in 347 biomedical research. Lab Anim Sci 47:337-445.

348 Guichelaar T, Hoeboer J, Widjojoatmodjo MN, et al (2014) Impaired immune response to vaccination 349 against infection with human respiratory syncytial virus at advanced age. $J$ Virol 88:9744-9750. 
Ichii $O$ et al. Glomerulus of aged cotton rats

351

352

353

354

355

356

357

358

359

360

361

362

363

364

365

366

367

368

369

370

371

372

Ichii O, Nakamura T, Irie T, et al (2016) Female cotton rats (Sigmodon hispidus) develop chronic anemia with renal inflammation and cystic changes. Histochem Cell Biol 146:351-362. https://doi.org/10.1007/s00418-016-1438-3

Ichii O, Nakamura T, Irie T, et al (2018) Close pathological correlations between chronic kidney disease and reproductive organ-associated abnormalities in female cotton rats. Exp Biol Med 243:418-427. https://doi.org/10.1177/1535370218758250

Ichii O, Yabuki A, Sasaki N, et al (2011) Pathological correlations between podocyte injuries and renal functions in canine and feline chronic kidney diseases. Histol Histopathol 26:1243-1255. https://doi.org/10.14670/HH-26.1243.

Iseki K, Ikemiya Y, Iseki C, Takishita S (2003) Proteinuria and the risk of developing end-stage renal disease. Kidney Int 63:1468-1474. https://doi.org/10.1046/j.1523-1755.2003.00868.x

Levey AS, Coresh J (2012) Chronic kidney disease. Lancet 379:165-180. https://doi.org/10.1016/S0140-6736(11)60178-5

Lim JH, Kim EN, Kim MY, et al (2012) Age-associated molecular changes in the kidney in aged mice. Oxid Med Cell Longev 2012:17138. https://doi.org/10.1155/2012/171383

Masakane I, Nakai S, Ogata S, et al (2015) An Overview of Regular Dialysis Treatment in Japan (As of 31 December 2013). Ther Apher Dial 19:540-574. https://doi.org/10.1111/1744-9987.12378.

Miner JH (2012) The glomerular basement membrane. Exp Cell Res 318:973-978. https://doi.org/10.1016/J.YEXCR.2012.02.031

Nakamura T, Ichii O, Irie T, et al (2018) Cotton rats (Sigmodon hispidus) possess pharyngeal pouch remnants originating from different primordia. Histol Histopathol 33:555-565. https://doi.org/10.14670/HH-11-946 
Nakamura T, Ichii O, Irie T, et al (2019) Cotton rat (Sigmodon hispidus) develops metabolic disorders associated with visceral adipose inflammation and fatty pancreas without obesity. Cell Tissue Res 375:483-492. https://doi.org/10.1007/s00441-018-2908-9 associated with survival in dogs with chronic kidney disease. J Vet Intern Med 32:1977-1982. https://doi.org/10.1111/jvim.15322 Silbiger SR, Neugarten J (1995) The impact of gender on the progression of chronic renal disease. Am J Kidney Dis 25:515-533. https://doi.org/10.1016/0272-6386(95)90119-1

381 Syme HM, Markwell PJ, Pfeiffer D, Elliott J (2006) Survival of cats with naturally occurring chronic 382 renal failure is related to severity of proteinuria. $J$ Vet Intern Med 20:528-535. https://doi.org/10.1111/j.1939-1676.2006.tb02892.x

Yang JW, Dettmar AK, Kronbichler A, Gee HY, Saleem M, Kim SH, Shin JI (2018) Recent advances of animal model of focal segmental glomerulosclerosis. Clin Exp Nephrol 22:752-763. https://doi.org/10.1007/s10157-018-1552-8 in renal glomeruli of mice. Exp Gerontol 24:237-249. https://doi.org/10.1016/0531-5565(89)90015-6 
Figure legends

393

Fig. 1 Hematologic parameters of cotton rats

a. Red blood cells (RBC).

b. Hematocrit (HCT).

c. Hemoglobin (Hgb).

d. White blood cells (WBC).

M: male; F: female; A.aged: advanced-aged group. ${ }^{*},{ }^{* *},{ }^{* * *}$ : significance with A.aged group $(P<$ 0.05, 0.01, 0.001). \#\#, \#\#: significance with males in the same groups $(P<0.01,0.001) . \dagger+\dagger$ : significance with old-aged females $(P<0.001)$.

e. Histopathologic features of the corticomedullary junction in cotton rats. Bars $=500 \mu \mathrm{m}$.

Fig. 2 Renal function parameters of cotton rats

a. Blood urea nitrogen (BUN).

\section{b. Serum creatinine (sCr).}

M: male; F: female. A.aged: advanced-aged group. *: significance with A.aged group $(P<0.05)$. \#\#\#: significance with males in the same groups $(P<0.001)$. $\dagger \dagger \dagger$ : significance with old-aged females $(P<0.001)$.

c. Urinary albumin. Urinary protein in the males was analyzed by SDS-PAGE. The strong bands detected are approximately $66 \mathrm{kDa}$ in size, similar to that of bovine serum albumin (BSA). $\mathrm{m}$ : 

stronger than those of young or adult males (3-5 m).

Fig. 3 Glomerular pathology of cotton rats

a. Young male. The glomerulus shows normal structures. Bar $=30 \mu \mathrm{m}$.

b. Advanced-aged (A.aged) female. The glomerulus structures are apparently normal, and cell (GBM) are noted. Bars $=30 \mu \mathrm{m}$.

e and f. A.aged male. Along with the GBM, PAS- or PAM-positive large granules or projections toward the podocytes are evident. Bars $=15 \mu \mathrm{m}$.

Fig. 4 Immune-complex deposition and podocyte molecules in the glomerulus of cotton rats

a. Immunofluorescence assay of IgG and IgA in young male cotton rats. No immune-positive reactions were observed for IgG or IgA. Blue: nuclear Hoechst staining. Bars $=50 \mu \mathrm{m}$. 

magnified area. Green: IgG- or IgA-positive reaction. Blue: nuclear Hoechst staining. Bars $=50 \mu \mathrm{m}$. $\mu \mathrm{m}$. projections of the GBM toward the podocytes are observed (arrows). Bars $=30 \mu \mathrm{m}$.

c and d. Irregular thickness of the GBM. Thinned or thickened GBMs are observed. In the latter case, large projections are also noted (arrows). Bars $=30 \mu \mathrm{m}$. regions (arrowheads) and reticular patterns of the GBM (asterisks) are observed. Pod: podocytes. Cap: capillary. Bars $=30 \mu \mathrm{m}$. 
Fig.1

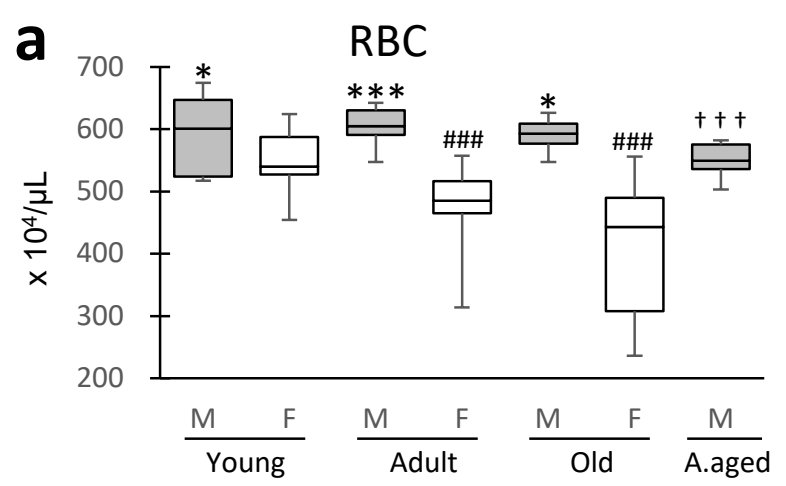

b
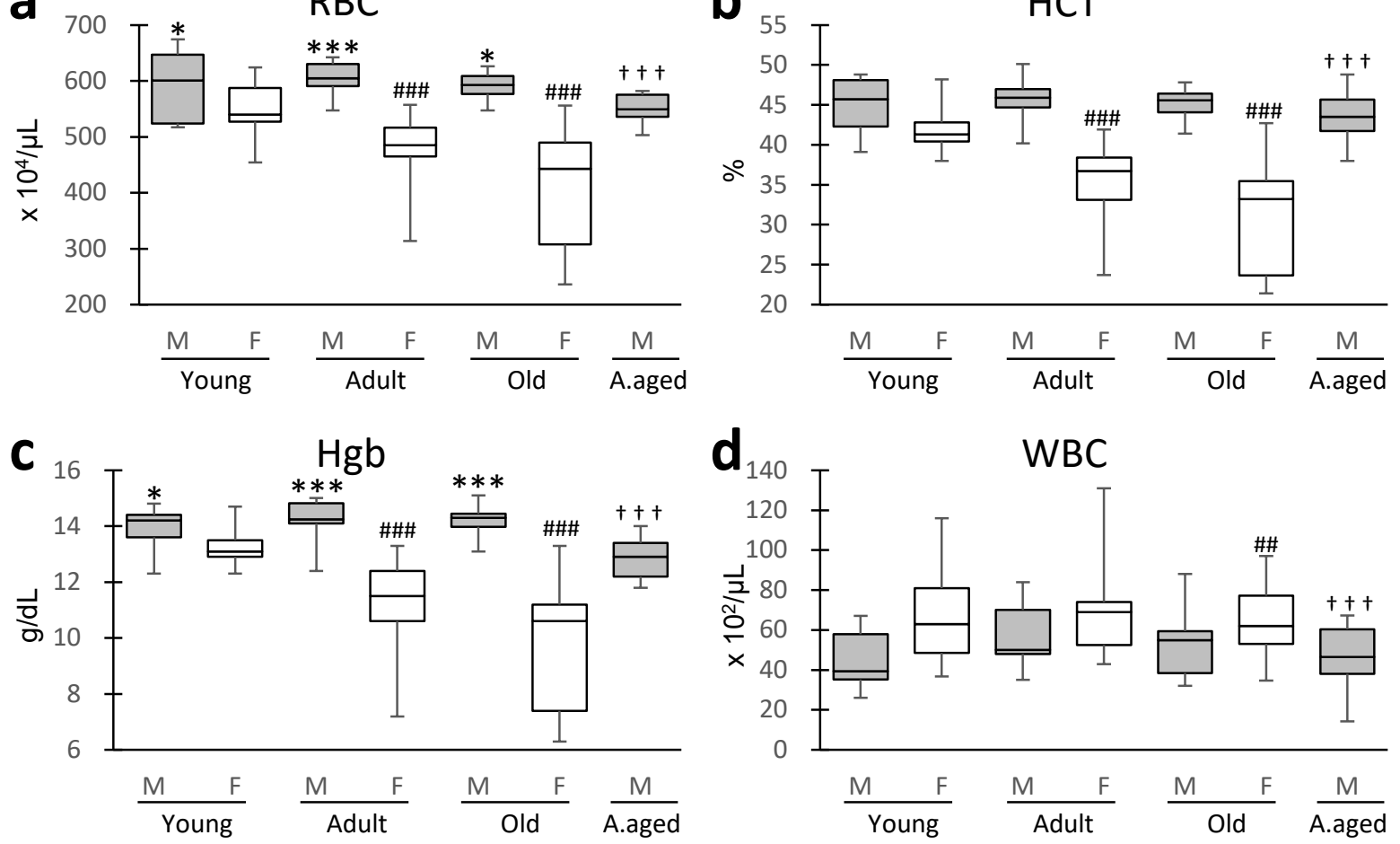

d

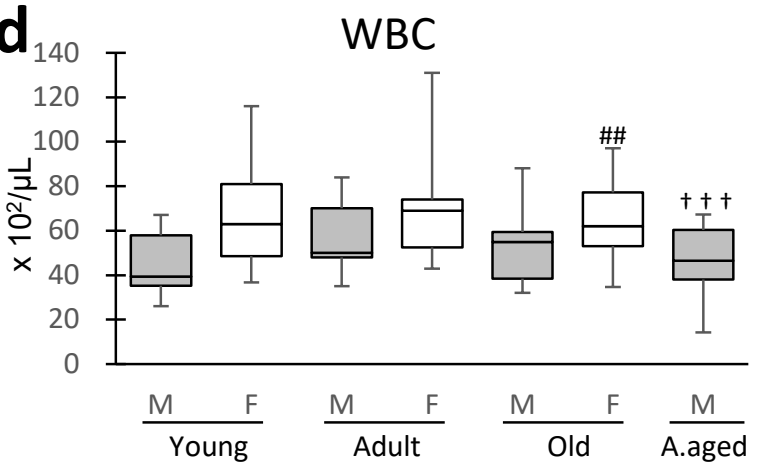

e

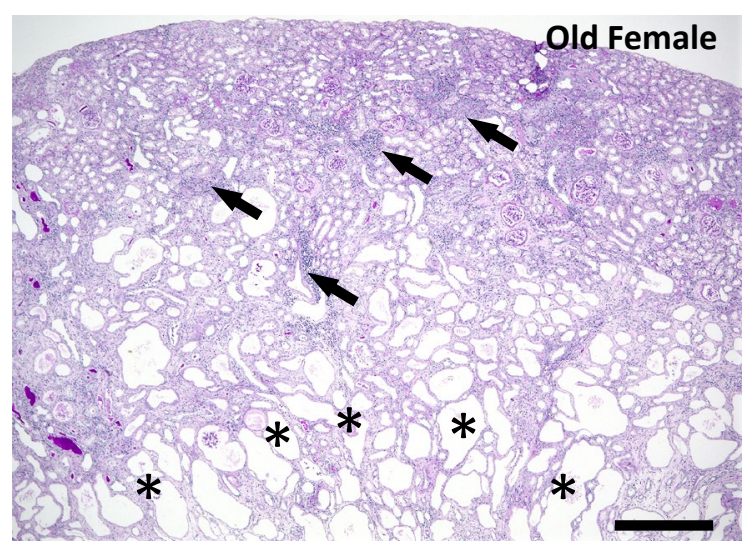

A.aged Male

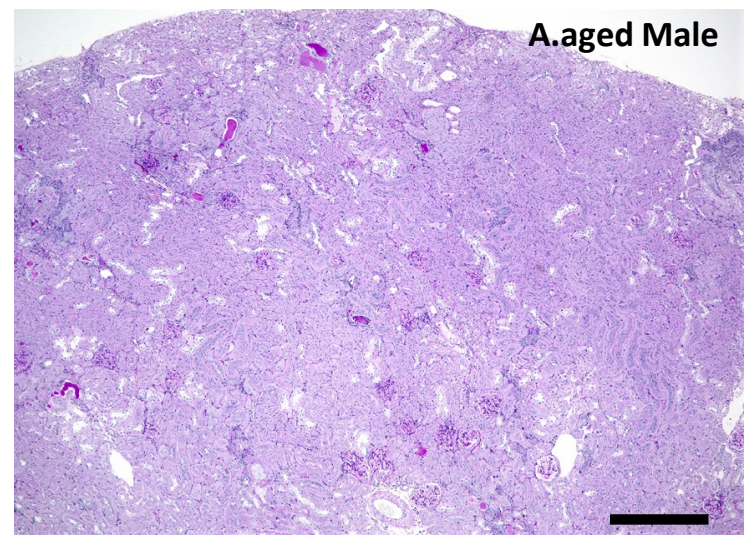


Fig. 2
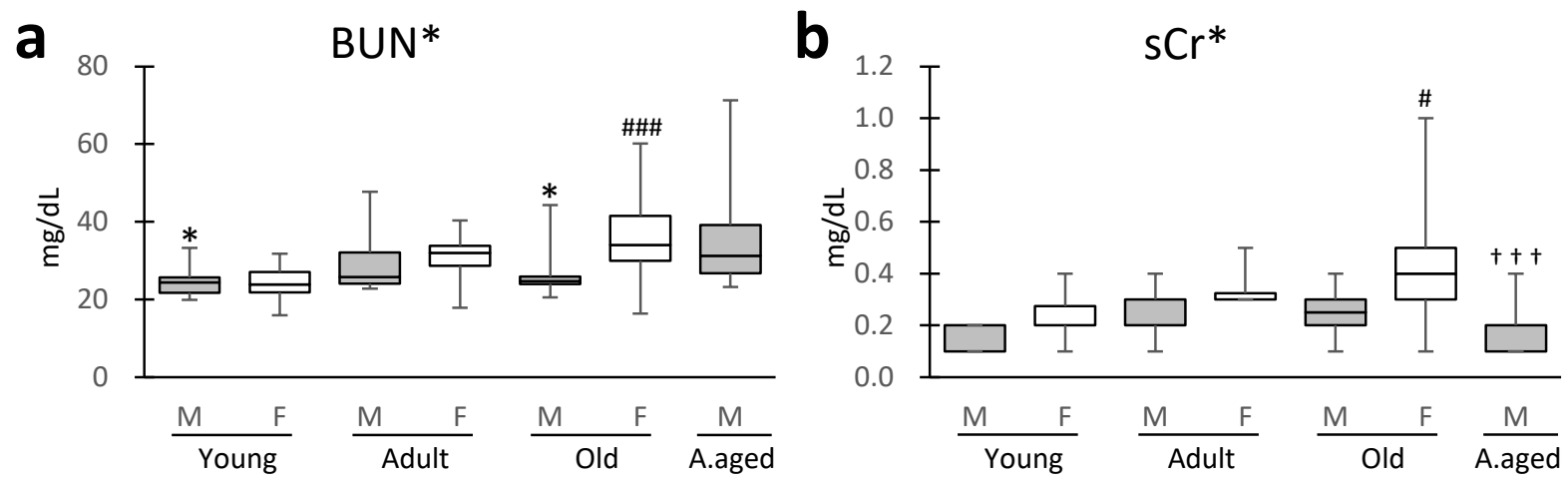

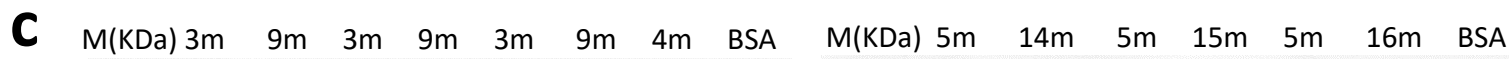

$97 \longleftarrow$

66

45
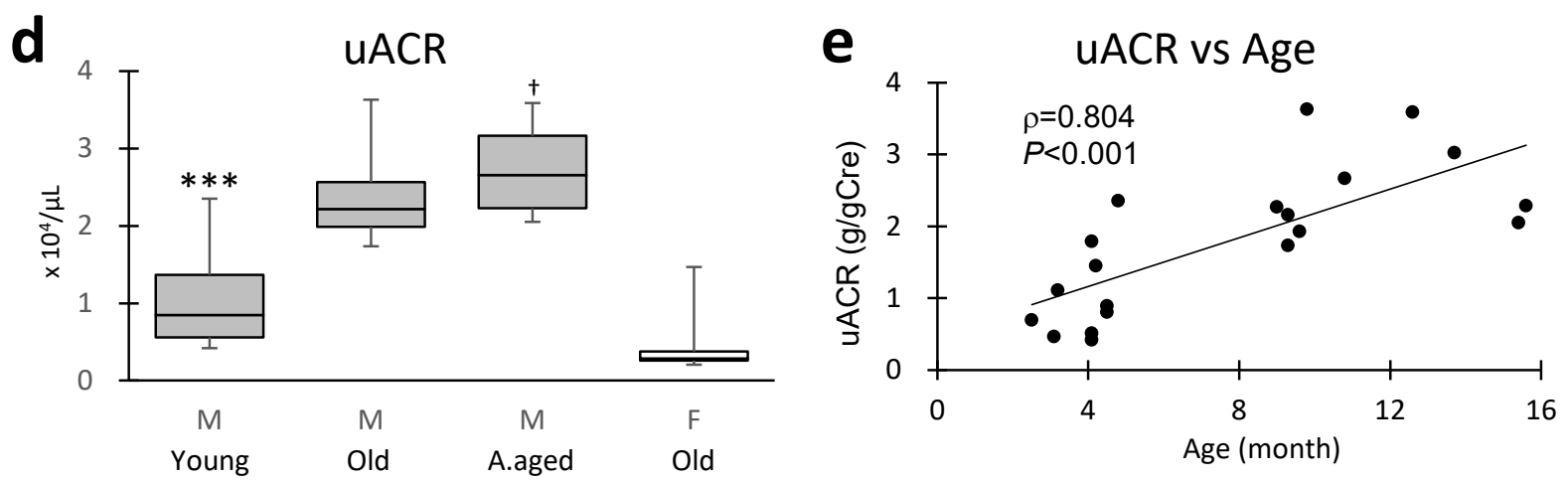
Fig.3
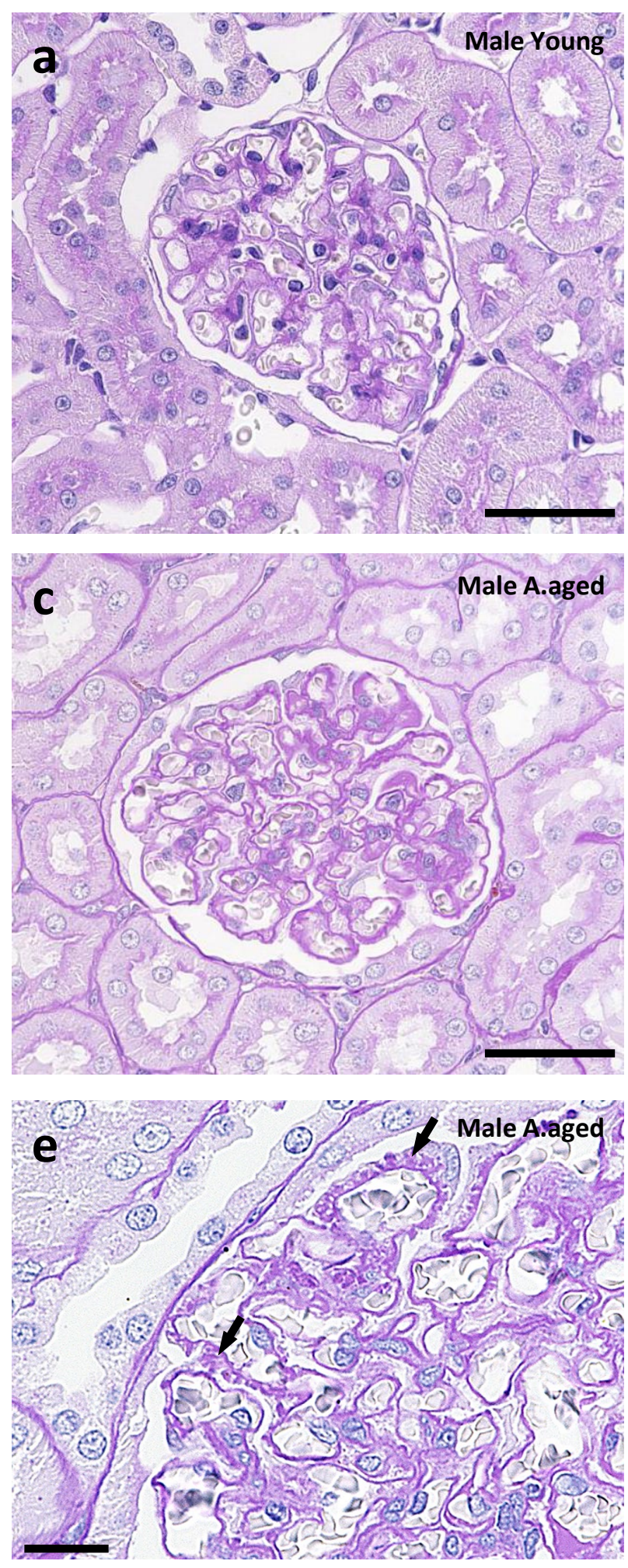
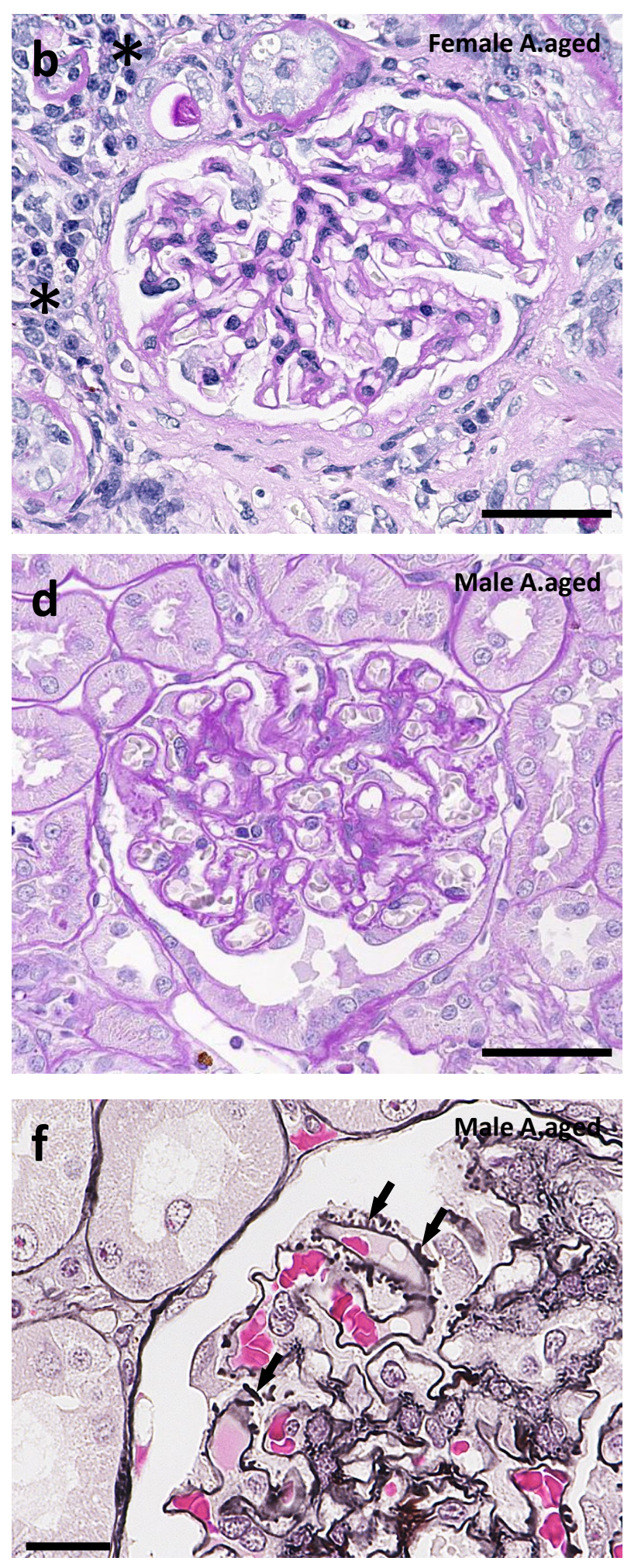
Fig.4
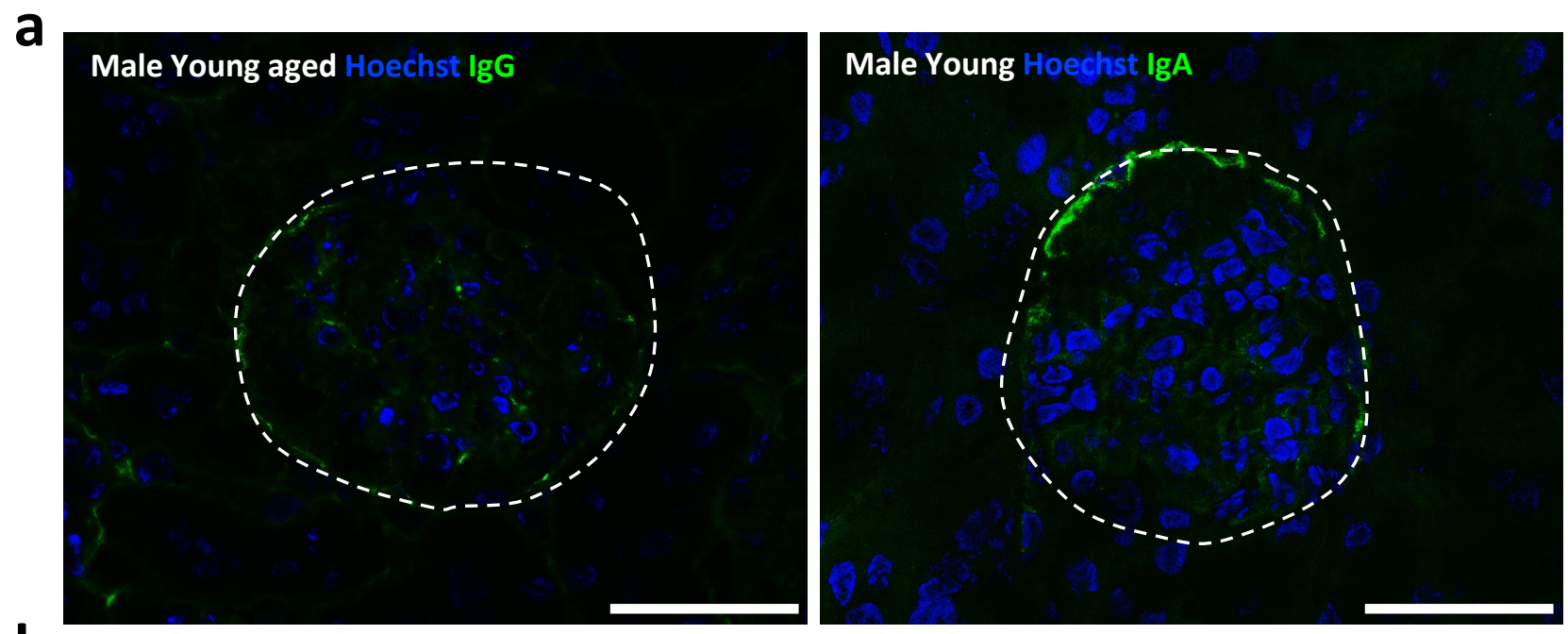

b
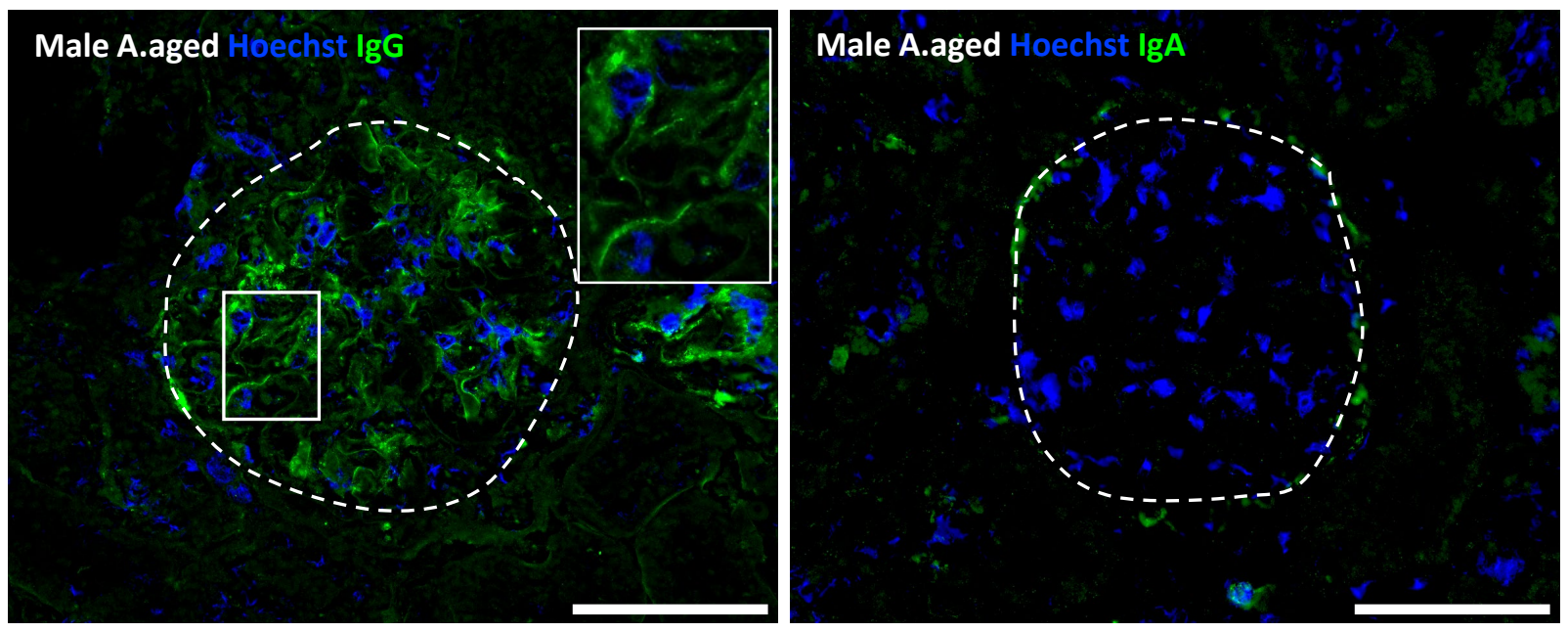

C

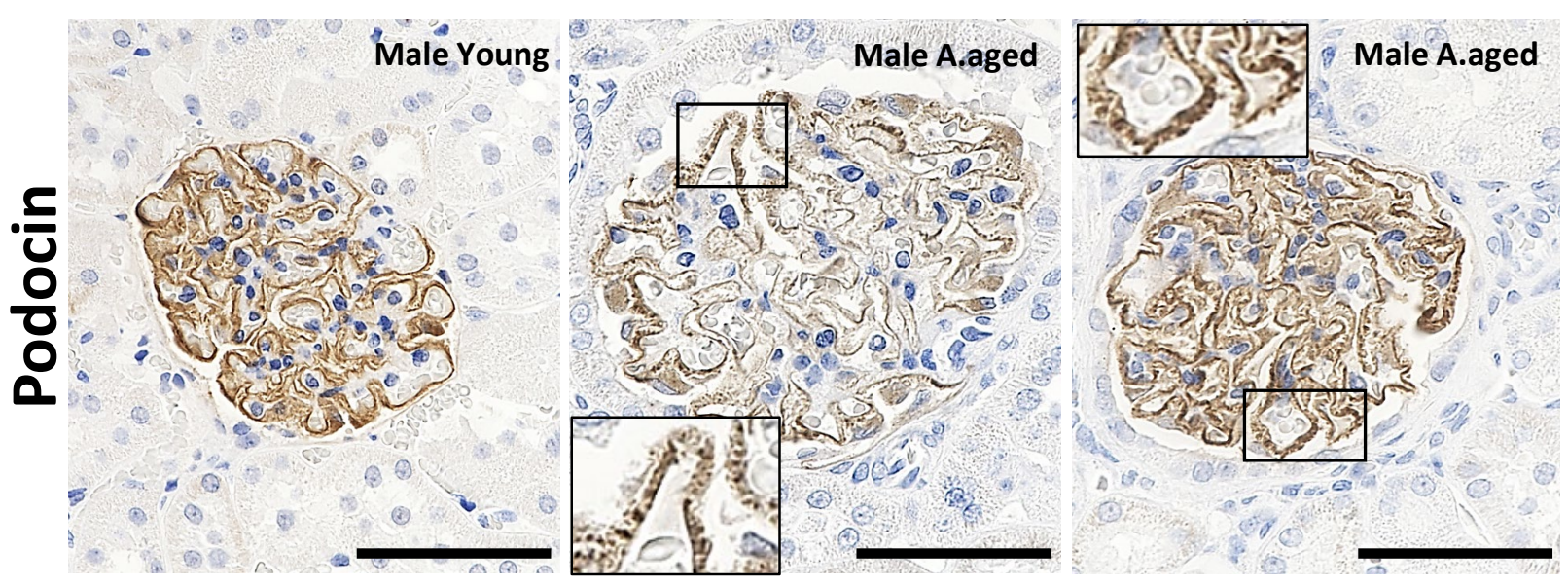


Fig.5
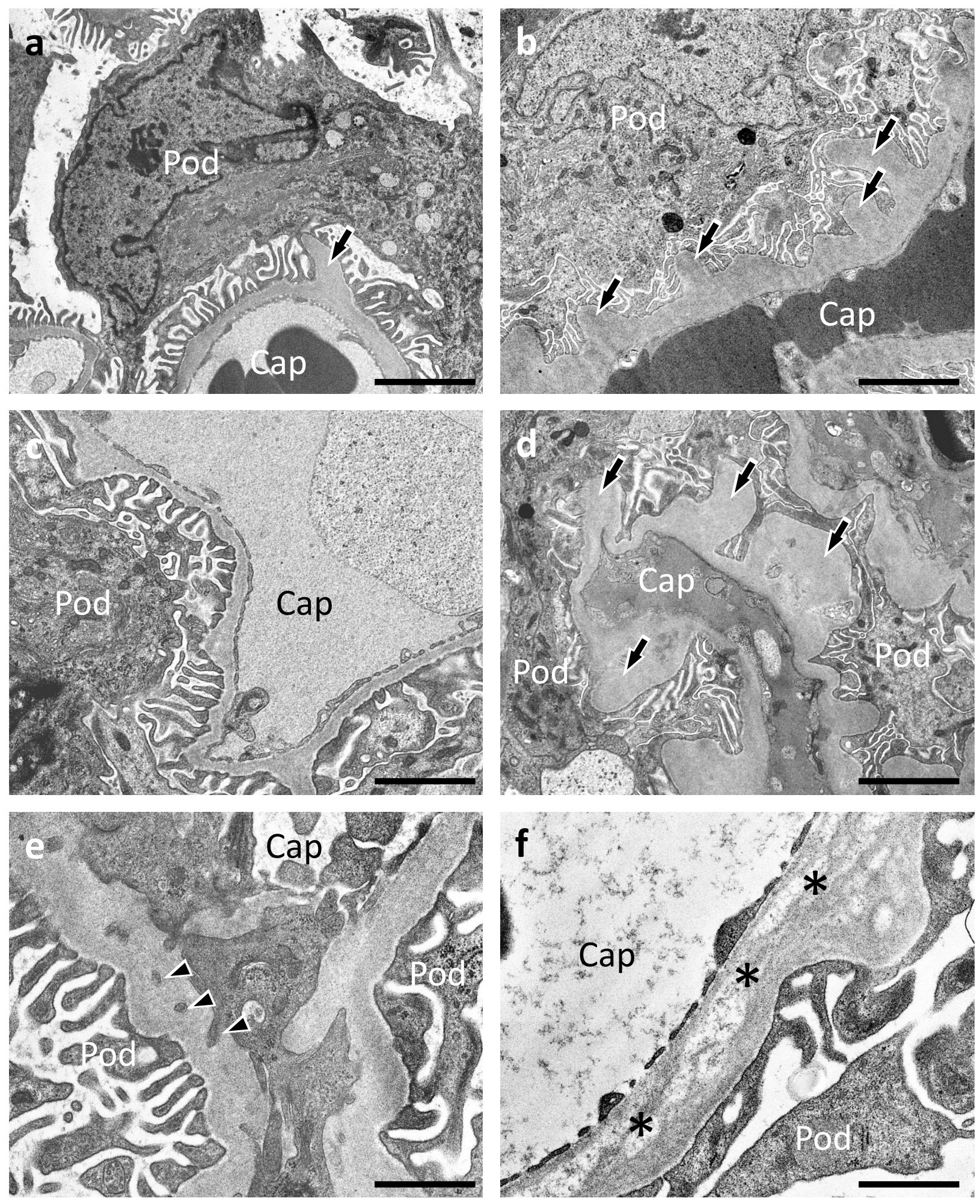
Supplemental Fig. 1
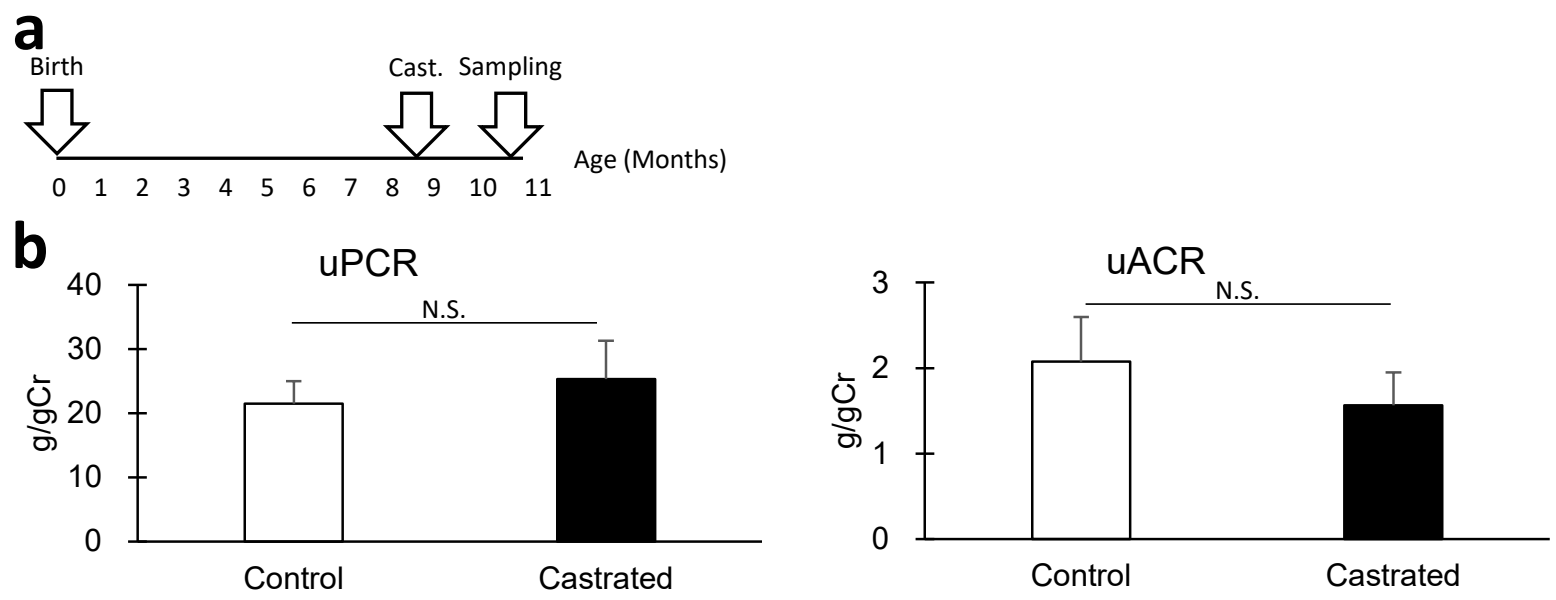